\title{
The Effects of Aerobic Exercise on Lipid Profile and Body Composition in Women With Multiple Sclerosis
}

\author{
Akram Monazamnezhad ${ }^{1, *}$; Abdolhamid Habibi ${ }^{2}$; Saeid shakeriyan ${ }^{2}$; Nastaran \\ Majdinasab ${ }^{3}$; Akbar Ghalvand ${ }^{1}$ \\ ${ }^{1}$ School of Physical Education and Sport Sciences, Shahid Chamran University, Ahvaz, IR Iran \\ ${ }^{2}$ Department of Sport Physiology, School of Physical Education and Sport Sciences, Shahid Chamran University, Ahvaz, IR Iran \\ ${ }^{3}$ Department of Neurology, School of Medicine, Ahvaz Jundishapur University of Medical Sciences, Ahvaz, IR Iran \\ ${ }^{*}$ Corresponding author: Akram Monazamnezhad, School of Physical Education and Sport Sciences, Shahid Chamran University, Ahvaz, IR Iran, E-mail: monazamnezhad.a@gmail. \\ com
}

Received: July 5, 2014; Revised: September 13, 2014; Accepted: November 10, 2014

\begin{abstract}
Background: Recent studies show that serum lipid profile and body composition have effects on the prognosis of multiple sclerosis (MS). Objectives: The purpose of this study was to investigate the effect of aerobic dancing training on serum lipid profile in women with RRMS (relapsing remitting multiple sclerosis).

Patients and Methods: Twenty-eight RRMS patients, age range 20-45 years with mild disability (expanded disability status scale 3 (EDSS 3)) participated in this semi-experimental study. The subjects were randomly divided into two groups: exercise group $(n=15)$ and control group $(\mathrm{n}=13)$. Lipid profile and body composition parameters were measured before and after the intervention. Exercise group participated in aerobic dancing training program at 50\%-70\% heart rate reserve (HRR) for 8 weeks, 3 sessions per week.

Results: At the end of the 8 week period, significant decreases have been found in TG (triglyceride), TC (total cholesterol), VLDL (very low density lipoprotein) levels and percent body fat $(\mathrm{PBF})$ in exercise group $(\mathrm{P}<0.05)$. Also, a significant difference observed in TG, VLDL levels, and PBF between 2 groups at the final measuring $(\mathrm{P}<0.05)$. There was no significant change in the investigated parameters between initial and final measurements in the control group $(\mathrm{P}>0.05)$.

Conclusions: In general, the results confirm the positive influence of the regular aerobic dance training on changes in lipid profile and body composition parameters in female patients with RRMS.
\end{abstract}

Keywords:Multiple Sclerosis; Body Composition; Exercise

\section{Background}

Multiple sclerosis (MS) is a chronic and debilitating disease of nervous system that destroys myelin of the central nervous system (brain and spinal cord) and gradually parts of the muscles lose their ability (1). As, the age of the disease onset ranges from 20 to 40 years, it can damage the productive forces of the society. According to the conducted researches, over 2.5 million adults suffer from MS worldwide and the number of patients is increasing day by day (2). Unfortunately, up-to-date statistics of MS disease in our country shows an upward trend. According to the report of Iranian Multiple Sclerosis Society, there are more than 40000 patients with MS in Iran. However, it is estimated that more than 50000 individuals are affected with MS (3). Also, based on the report of Khuzestan Multiple Sclerosis Society, 735 patients with MS are residents of Ahvaz in October 2013.

The etiology of MS is unknown, but many factors are associated with its prognosis. Among these factors, feeding habits and body composition are especially important and these topics have been studied recently (4). Weinstock-Guttman et al. investigated the associations of serum lipid profile with disability levels (EDSS) and MRI measures. They have demonstrated that the lipid profile can adversely affect MS progression, particularly higher LDL-C (low density lipoprotein-cholesterol) and TC (total cholesterol) and lower HDL-C (High density lipoproteincholesterol) levels are associated with more inflammatory activity in MS patients $(5,6)$. Also, it was observed that plasma lipid profile and body fat percentage are associated with cardiovascular diseases progression (7). Some studies reported that triglyceride and cholesterol levels of MS patients were higher compared to healthy subjects of similar age and sex. Moreover, the prevalence of cardiovascular disease in this population is higher $(4,8)$. Recently observed cardiovascular disease due to dyslipidemia is associated with more rapid disability progression in multiple sclerosis $(9,10)$. Furthermore, inactivity or reduced physical activity level in these patients can be the result of physical disability, imbalance, motor problems, and fatigue that lead to increased cholesterol and triglyceride levels. On the other hand, exercise not only reduces total cholesterol (TC), but also can increase high density

Copyright (C) 2015, Ahvaz Jundishapur University of Medical Sciences. This is an open-access article distributed under the terms of the Creative Commons Attribution-NonCommercial 4.0 International License (http://creativecommons.org/licenses/by-nc/4.0/) which permits copy and redistribute the material just in noncommercial usages, provided the original work is properly cited. 
lipoprotein (HDL) and decrease low density lipoprotein (LDL) (11). Triglyceride is a fat that is available to use and even low-intensity exercise can convert it to fatty acids. Total cholesterol is a combination of lipids that is used to provide energy by exercise with determined intensity and duration. Released fatty acids from adipose tissue into blood and muscle triglycerides are the main sources of energy during exercise (12). Evidence shows that the increased secretion of catecholamines and growth hormone during physical activity leads to increase in lipolysis. Therefore, using fat stores as an energy source increases during physical activity.

Also, other mechanisms like decreased insulin sensitivity that can change blood lipids and lipoprotein levels may be effective $(13,14)$. The relationship of specific anthropometric features and body type, on one hand, and the possibility of some disease, on the other hand, has been approved. Behaeein et al. investigated the relationship between body type and types of MS (relapsingremitting, secondary progressive, primary progressive) in females. The finding of their study indicated that MS women with relapsing-remitting (RR) had higher fat mass and average weight in comparison with those of other types of MS and healthy subjects. The RR group was more endomorphic (15). Recently published data show that the energy cost of walking in mildly disabled individuals with MS is higher than healthy subjects (16). If reduced physical activity and probably reduced energy expenditure in MS are not accompanied by a reduction in energy intake, body fat will increase (17). There are many detrimental manifestations of excess body fat, such as hyperlipidemia, insulin resistance, and type 2 diabetes (16). Thus, MS individuals with greater body fat are at a greater risk for these diseases that may be related to unfavorable changes in body composition that result from lack of physical activity and neuromuscular problems (17). Body composition assessment is important in order to determine an ideal body composition for healthy livings. Since BMI is associated with body fat, it is the most common indicator of obesity that can change with age, sex, lifestyle, and disease (18). Not many studies have investigated body mass index (BMI) in patients with MS. It was observed that in these patients both total body fat and fat free mass showed consistent dependence on BMI, as among normal subjects (16).

Evidence suggests that physical activity and exercise are the most important factors for improving physical symptoms of MS. For several years, patients with MS were advised not to participate in exercise because it was reported to worsen symptoms or lead to fatigue. The findings of the studies conducted during the last two decades showed that people with MS receive substantial benefit from physical activity. Exercise has been shown to be safe and very well tolerated among people with MS $(19,20)$. Exercise program for people with MS should be based on the patient's degree of disability. For example, MS patients with mild degree of disability are able to perform physi- cal activity independently (21). In this regard, one of the most popular programs is aerobic dancing. This program is popular because of using enjoyable exercises with music, also the selected exercise routine is easy for everyone. The participants in this study had EDSS $\leq 3$ and were able to walk independently, also based on the 6-minute walk test that used to assess aerobic and functional capacity in the pretest and with regard to aerobic exercise effects on lipid profile and body composition (22-24).

\section{Objectives}

The present study investigated the effects of rhythmic aerobic on lipid profile and body composition in sedentary females with multiple sclerosis.

\section{Patients and Methods}

The aim of this semi-experimental study is to investigate the effect of aerobic exercise on body composition and lipid profile in women with MS. According to the previous similar studies (12), sample size formula, and the recommendation of the statistical advisor, the sample size was considered at 30 patients. The population of this research included female MS patients who were members of Ahvaz MS Society. Thirty volunteers with RRMS who met the inclusion criteria were selected and classified randomly into 2 groups (exercise and control). Among subjects in the control group two patients were excluded from the study because of failure to complete the study. Finally, 28 patients completed the study (exercise group: 15 and control group: 13). Inclusion criteria were as follows : women with relapsing-remitting MS, aged 20-45 years, EDSS $\leq 3$, no history of other neurologic, metabolic, orthopedic, cardiovascular, respiratory diseases, blood pressure, diabetes, or other medical condition that would preclude their participation in the training, performing 6-minute walk test (6 MWT) independently, no regular participation in a regular aerobic exercise program within 6 months prior to the study, non-smoker and non-alcohol user, no history of exacerbation within 6 months prior to study. Exclusion criteria were as follows: resignation or absence from exercise for more than 2 consecutive sessions, regular exercise in the control group, participating in exercise sessions except the present study, relapsing during exercise or hospitalization, taking statins and corticosteroids (All of the patients were treated with interferons). First, a neurologist evaluated physical health of patients and expanded disability status Scale or EDSS score. This questionnaire assesses the state and functions of the central nervous system, including pyramidal functions, cerebellar function, brain stem function, sensory function, bowel and bladder functions, visual or optical functions, cerebral or mental function. EDSS score ranges on a scale from 0 to 10, with higher scores indicating more severe disability (25). Initially, all subjects participated in an orientation session and they were given informa- 
tion about the study protocol, possible side effects, and safety of the exercise training. During the first session, the participants completed a medical history questionnaire, demographics scale and signed the informed consent form. The protocol and all the procedures were carried out in accordance with the Helsinki Declaration and the international principles governing research with human beings.

Body weight (BW), body mass index (BMI), and body fat percentage (BFP) were measured by body composition analyzer machine (Olympic Model 3/3, made in Korea). Patients in the fasting state referred to the physiology laboratory of Chamran University. The machine had places for feet and two bars for palm and fingers contact. The output data were printed on a special form through a printer connected to the analyzer. In this study, subjects were MS patients with mild degree of disability. Therefore, we evaluated their ability to walk independently and their aerobic capacity (endurance) with 6 MWT according to the standard protocol. The subjects walked around the basketball court for 6 minutes and the distance traveled by them was calculated. Two days before the first training session and 2 days after the last training session, blood sample was taken after 10 to 12 hours of fasting, between 9:00 and 10:00 AM. Serum and red blood cells were separated by centrifugation and serum was used for measuring lipid profile. serum total cholesterol(TC), high density lipoprotein (HDL) and triacylglycerol (TG) were measured before and after study by photometric method using Pars Azmoon kits in a national biochemical laboratory (Lab Golestan, Ahvaz, Iran). Low density lipoprotein was estimated using Friedewald formula (26).

In this study, the intervention was rhythmic aerobic training program that patients performed under supervision of the researchers. Training program was started at $50 \%$ of Heart rate reserve (HRR) at the first week and increased to $70 \%$ of HRR at the eight week.

The Heart rate reserve (HRR) was determined by Karvonen Formula (27).

Maximal heart rate $=220$ - Age

Heart rate reserve $(\mathrm{HRR})=$ Maximal heart rate $(\mathrm{HR}$ max) - Resting heart rate (HR rest)

Target heart rate $=(50 \%-70 \%$ HRR $)+$ HR rest.

During each session the subjects' heart rate was monitored by manual palpation from the carotid artery to control the heart rate responses. Counting the beats by manual palpation from carotid artery is easy during movement (28). The subjects were given information about the manual palpation heart rate method from the carotid artery during the orientation session. Each session consisted of 10 minutes of warming up, including fast walking, jogging, and dynamic stretch. Workout included rhythmic aerobic exercise with brief resting periods. The length of workout increased gradually from 2030 minutes. Rhythmic aerobic comprises coordinated movements of feet and hands. Some of the movements consisted of stretching exercises, the "step-touch", "sideto-side", "V-step", and walking back and front. Choreographed sequences consisted of various combinations of the aforementioned steps with the addition of hand and body movements used to increase intensity. At the end of each session, 10 minutes of cooling down was performed for recovery that included static stretch in sitting position. The subjects in exercise group based on their training protocol participated in three 45-minute session per week for 4 weeks initially, followed by three 60-minute session per week for another 4 weeks. The control group consumed their prescription drugs and continued their routine life.

\subsection{Statistics}

SPSS 19.0 software was used for analyzing data. The data of the subjects before and after 8-week training were compared and analyzed between training with Paired and independent samples t test. Significant levels in all tests were considered at $\mathrm{P} \leq 0.05$.

\section{Results}

Demographic characteristics of the participants are summarized in Table 1. Tables 2 and 3 indicate data analysis of two groups. Results revealed significant differences from pretest to posttest measurements in the exercise group regarding TG, TC, VLDL, and BFP $(\mathrm{P}<0.05)$. In the control group, there were no significant changes in all variables $(\mathrm{P}>0.05)$. Also, there were significant differences between the exercise and control groups with regard to TG, VLDL, and $\mathrm{BFP}(\mathrm{P}<0.05)$.

Table 2 presents the comparative values of lipid profile and body composition in two groups before and after intervention. TG $(\mathrm{P}=0.019), \mathrm{TC}(\mathrm{P}=0.037)$, VLDL $(\mathrm{P}=$ $0.019)$, and $B F P(P=0.013)$ showed significant changes in exercise group, whereas no significant change was observed in the control group.

The comparison of differences in parameters between 2 groups is shown in Table 3. According to this table, TG $(\mathrm{P}=0.007)$, VLDL $(\mathrm{P}=0.008)$, and $\mathrm{BFP}(\mathrm{P}=0.007)$ showed significant changes. However, differences in TC, HDL, LDL, BW, and BMI levels between the exercise and control groups were not statistically significant $(\mathrm{P}>0.05)$.

\begin{tabular}{|c|c|c|}
\hline Variable & $\begin{array}{l}\text { Exercise group } \\
\quad(\mathbf{n}=15)\end{array}$ & $\begin{array}{c}\text { Control group } \\
(\mathbf{n}=13)\end{array}$ \\
\hline Age, y & $36.07 \pm 5.91$ & $34.08 \pm 6.94$ \\
\hline Height, cm & $162.73 \pm 6.36$ & $163.30 \pm 7.22$ \\
\hline Body weight, kg & $68.06 \pm 13.32$ & $69.26 \pm 11.53$ \\
\hline BMI, $\mathrm{kg} / \mathrm{m}^{2}$ & $25.56 \pm 3.54$ & $26.03 \pm 4.39$ \\
\hline
\end{tabular}

\footnotetext{
a Abbreviation: BMI, body mass index.

$\mathrm{b}$ Data are presented as Mean \pm SD
} 
Monazamnezhad A et al.

Table 2. Preintervention and Postintervention Values for Triglyceride, Total Cholesterol, Low-Density Cholesterol, High-density Cholesterol, and Very Low-density Cholesterol ${ }^{\text {a }}$

\begin{tabular}{|c|c|c|c|c|c|c|}
\hline \multirow[t]{2}{*}{ Variable } & \multicolumn{3}{|c|}{ Control Group } & \multicolumn{3}{|c|}{ Exercise Group } \\
\hline & Pretest & Posttest & P Value & Pretest & Posttest & PValue \\
\hline TG, mg/dL & $126.46 \pm 35.84$ & $130.85 \pm 33.99$ & 0.205 & $119.93 \pm 36.58$ & $103.67 \pm 24.79$ & $0.019^{b}$ \\
\hline $\mathrm{TC}, \mathrm{mg} / \mathrm{dL}$ & $186.62 \pm 21.23$ & $185.85 \pm 21.91$ & 0.697 & $180.67 \pm 26.69$ & $169.67 \pm 15.29$ & $0.037^{b}$ \\
\hline LDL, mg/dL & $110.16 \pm 21.31$ & $109.36 \pm 21.02$ & 0.751 & $107.41 \pm 29.28$ & $99 \pm 17.34$ & 0.159 \\
\hline HDL, mg/dL & $51.15 \pm 10.34$ & $50.31 \pm 8.98$ & 0.631 & $49.27 \pm 9.84$ & $50.40 \pm 8.72$ & 0.282 \\
\hline VLDL, mg/dL & $25.29 \pm 7.16$ & $26.16 \pm 6.79$ & 0.205 & $23.98 \pm 7.31$ & $20.73 \pm 4.95$ & $0.019^{b}$ \\
\hline BW, kg & $69.26 \pm 11.53$ & $69 \pm 11.06$ & 0.495 & $68.06 \pm 13.32$ & $66.90 \pm 11.96$ & 0.062 \\
\hline BMI, $\mathrm{kg} / \mathrm{m}^{2}$ & $26.03 \pm 4.39$ & $25.94 \pm 4.26$ & 0.535 & $25.56 \pm 3.54$ & $25.15 \pm 3.16$ & 0.077 \\
\hline BFP,\% & $32.27 \pm 4.58$ & $32.39 \pm 4.44$ & 0.393 & $31.69 \pm 3.60$ & $30.77 \pm 3.24$ & $0.013^{b}$ \\
\hline
\end{tabular}

${ }^{a}$ Abbreviations: BMI, body mass index; BFP, body fat percent; BW, body weight; HDL, high-density lipoprotein cholesterol; LDL, low-density lipoprotein cholesterol; TG, triglyceride; TC, total cholesterol; VLDL, very low-density cholesterol.

$\mathrm{b}$ Significant difference between preintervention and postintervention at $\mathrm{P}<0.05$.

Table 3. Comparison of Mean Difference of Measured Variables During 8 Weeks between Exercise and Control Groups

\begin{tabular}{lccc}
\hline Variable & Control Group & Exercise Group & P Value \\
\hline TG, $\mathbf{~ m g / d L ~}$ & $4.38 \pm 11.79$ & $-16.26 \pm 23.68$ & $0.007^{\mathrm{a}}$ \\
$\mathbf{T C}, \mathbf{m g} / \mathbf{d L}$ & $-0.76 \pm 6.95$ & $-11.00 \pm 18.46$ & 0.062 \\
\hline $\mathbf{L D L}, \mathbf{m g} / \mathbf{d L}$ & $-0.80 \pm 8.87$ & $-8.41 \pm 21.87$ & 0.232 \\
$\mathbf{H D L}, \mathbf{m g} / \mathbf{d L}$ & $-0.84 \pm 6.18$ & $1.13 \pm 3.92$ & 0.315 \\
VLDL, $\mathbf{m g} / \mathbf{d L}$ & $0.87 \pm 2.35$ & $-3.25 \pm 4.73$ & $0.007^{\mathrm{a}}$ \\
$\mathbf{B W}, \mathbf{m g} / \mathbf{d L}$ & $-0.26 \pm 1.37$ & $-1.16 \pm 2.22$ & 0.206 \\
$\mathbf{B M I}, \mathbf{k g} / \mathbf{m}^{2}$ & $-0.09 \pm 0.52$ & $-0.40 \pm 0.82$ & 0.234 \\
$\mathbf{B F P}, \%$ & $0.12 \pm 0.51$ & $-0.92 \pm 1.25$ & $0.008^{\mathrm{a}}$ \\
\hline
\end{tabular}

a Significant difference between exercise and control groups at $\mathrm{P}<$ 0.05 .

\section{Discussion}

Because of the negative impact of blood lipid and body composition abnormalities in progression of MS, this study investigated the effect of 8 weeks of aerobic exercise on lipid profile and body composition in women with RRMS. It has been thought that MS can alter the lipid metabolism and body fat storage and distribution (29, 30 ). The increasing production of interleukin-1 might increase plasma triglycerides and LDL and causes abnormal fat distribution by inhibiting lipoprotein lipase activity in patients with MS. Also, demyelination can be another reason for hyperlipidemia (2). It was demonstrated that worsening of disability as assessed by the Extended Disability Status Scale and the MS Severity Scale was associated with higher baseline low-density lipoprotein cholesterol and total cholesterol levels $(5,31)$. These findings indicate the importance of aerobic and physical activity to improve lipid and lipoprotein levels in patients with MS.

So far, few studies (with conflicting results) have examined the effects of exercise on lipid profile and body com- position in patients with MS. Petajan et al. have found a significant decrease in TG after 15 weeks combined arm and leg ergometry (32). The seminal study by Schapiro et al. reported a significant decrease in TG level after a period of aerobic exercise (33). These results are consistent with our finding for triglyceride. Some of the effective adaptations induced by aerobic exercise are increased mitochondria and subsequently increased lipolysis enzymes activity that result in an increase in lipids catabolism during exercise (34). Change in LPL activity results in more influx of VLDL into the circulation from the liver, which will be removed from the circulation (35). Also, increasing LPL activity leads to conversion of VLDL to HDL, and subsequently a decrease in VLDL and an increase in HDL level (13). Exercise may indirectly influence lipids by reduction in abdominal fat, and subsequently release of free fatty acids (FFA) from abdominal fat into liver decreases, which leads to reduction in hepatic VLDL production (35). Furthermore, hepatic lipase decreases with long and regular physical activity (36). Other possible mechanisms such as reduction in body fat percent (BFP) may play a role in reducing cholesterol and triglyceride (12). Our results for lipid profile parameters are inconsistent with Marsh research (37). Perhaps this is due to differences in the training period that was 4 weeks in Marsh study or differences in the subjects' physiologic conditions, nutrition, energy cost, or lower exercise intensity in Marsh study. It was demonstrated that lipid profile changes is associated with changes in fat mass (38). Our findings show that aerobic training has a significant effect on body fat percentage in MS patients. Sedentary lifestyle adopted by MS patients leads to increase body fat percentage and leptin hormone, which is associated with coronary diseases (39). Some researchers suggest that leptin is a warning hormone for body fat regulation. Cooperation of leptin and insulin can influence sympathetic nervous system and vascular function. Vascular dysfunction is associated with increased leptin (40). Recent studies have shown that physical activity, aerobic training, especially leads 
to a significant decrease of leptin levels. Reduction in leptin levels can decrease body fat percentage that leads to increase in aerobic capacity, mobility and balance ability in MS patients (41). There have been only few studies researching BFP in MS patients. The results of this study indicate that aerobic exercise is effective to reduce BFP in MS patients. It seems that aerobic exercise can stimulate lipolysis process that leads to decreased plasma levels of TG, TC, and BFP. Our results show a reduction in LDL and an increase in HDL levels after 8 weeks of aerobic training in the exercise group, but these changes were not statically significant. These results are consistent with Petajan et al., Schapiro et al., and Marsh et al. studies (32, 33, 37). It is possible, duration and intensity of the exercise training was not adequate to change LDL and HLD levels significantly. Also, baseline levels of these indicators at the start of the training period is an effective factor, so that abnormal lipid levels change more tangible than normal lipid levels in response to exercise (42). In this study, the baseline levels of the lipids and lipoproteins were in the high normal range. Some other possible reasons may be small sample size and the subjects' nutrition in our research. Most researchers and nutritionists suggest long-term and regular aerobic exercise training, in order to burn fat and use it as a source of energy. Furthermore, low fat diet (less than 30\%) is effective in reducing body weight (43). Nutrition plays an important role in TC and LDL levels. Our results showed that there was a slight but not significant decrease in TC and LDL levels of the control group, which indicates that the dietary intake of the control group might have changed during the study period. This confirms the important role of nutrition in lipid levels. In the present study, we investigated only the effect of exercise and had no control over the subjects' nutrition. In general, LDL and TC levels are lower in persons with aerobic physical activity that result from their lower body weight and body fat (44). The finding of the present study indicated a decrease in BMI and BW after 8 weeks in the exercise group. However, these reductions were not significant. It appears that a more long-term exercise training and monitored diet are needed to achieve a significant BW and BMI reduction. Several studies have shown that caloric restriction can improve body composition by reducing BW, BMI, and BFP. Also, physical activity has been shown to have a modest effect on body weight (45). Formica et al. have reported that fat-free mass (FFM) decreases in sedentary MS patients, which can be induced by skeletal muscle atrophy (46). Dietary restriction only is accompanied by decreased FFM that leads to reduction in basal metabolic rate (BMR). This can maintain fat reserves and slow weight loss (45). Therefore, maintaining lean body mass by exercise is an appropriate way to maintain long-term weight loss and fat-free mass, particularly in patients with MS. In this study, the patients had a sedentary lifestyle for fear of worsening the symptoms like fatigue. When the intervention starts the subjects participated in regular aerobic exercise and its positive effects on their lipid profile and body fat was observed at the end of the intervention. In general, our findings indicate favorable effects of aerobic exercise on body composition and lipid profile in women with MS. However, many factors such as patients' nutrition, exercise intensity, duration, and number of training sessions can affect the results. Due to the study limitations, we feel that there is a need to conduct similar studies with larger sample size, longitudinal training intervention, and controlled diet.

The findings of the study indicate that regular aerobic exercise can improve lipid profile and body composition in women with MS. The patients can benefit from aerobic exercise according to their abilities in addition to drug treatments. Therefore, MS patients who engage in aerobic exercise can prevent the progression of MS and secondary conditions.

\section{Acknowledgements}

This study is a part of MSc Thesis submitted by Akram Monazamnezhad, No: 92033270. The authors would like to thank Multiple Sclerosis Society of Ahvaz-Iran, all participants in the study, the staff of Physical Education and Sport Sciences faculty of Shahid Chamran University, and Rehabilitation faculty of Ahvaz Jundishapur University of Medical Sciences, Ahvaz, Iran.

\section{References}

1. Comi G, Leocani L, Rossi P, Colombo B. Physiopathology and treatment of fatigue in multiple sclerosis. J Neurol. 2001;248(3):174-9.

2. Ghasemi E, Shayegannejad V, Ashtari F, Moradi B, Rezaei E, Keivanfar N. An investigation on the effects of neuromuscular exercise on balance, gait and the depression of paitent with MS. $J$ Res rehabilit Sci. 2011.

3. Pazokian M, Shaban M, Zakerimoghdam M, Mehran A, Sanglaje $B$. The effect of stretching together aerobic exercises on fatigue level in multiple sclerosis patients refer to MS society of iran those suffer from fatigue. Holist Nurs Midwifery. 2012;22(2):18-24.

4. Comoglue S, Yardimci S, Okcu Z. Body fat distribution and plasma lipid profiles of patients with multiple sclerosis. Turk J Med Sci. 2004;34:43-8.

5. Weinstock-Guttman B, Zivadinov R, Mahfooz N, Carl E, Drake A, Schneider J, et al. Serum lipid profiles are associated with disability and MRI outcomes in multiple sclerosis. J Neuroinflammation. 2011;8:127.

6. Weinstock-Guttman B, Zivadinov R, Horakova D, Havrdova E, Ou J, Shyh G, et al. Lipid profiles are associated with lesion formation over 24 months in interferon-beta treated patients following the first demyelinating event. J Neurol Neurosurg Psychiatry. 2013;84(11):1186-91.

7. AACPR.. Guidelines for Cardiac Rehabilitation and Secondary Prevention Programs. 4th edHK: Champaign; 2004.

8. Dalgas U, Stenager E, Ingemann-Hansen T. Multiple sclerosis and physical exercise: recommendations for the application of resistance-, endurance- and combined training. Mult Scler. 2008;14(1):35-53.

9. Motl RW, Fernhall B, McAuley E, Cutter G. Physical activity and self-reported cardiovascular comorbidities in persons with multiple sclerosis: evidence from a cross-sectional analysis. Neuroepidemiology. 2011;36(3):183-91.

10. Marrie RA, Rudick R, Horwitz R, Cutter G, Tyry T, Campagnolo D, et al. Vascular comorbidity is associated with more rapid disability progression in multiple sclerosis. Neurology. 
2010;74(13):1041-7.

11. Stuifbergen AK, Blozis SA, Harrison TC, Becker HA. Exercise, functional limitations, and quality of life: A longitudinal study of persons with multiple sclerosis. Arch Phys Med Rehabil. 2006;87(7):935-43.

12. Khajei R, Soltani M, Hejazi M. , Noor Nematolahi S, Zendedel A Ashkanifar M. The Effect Of Aquatic Aerobics Exercises On Cardiovascular Risk Factors In Patients With Multiple Scleroesis. Evidence Based Care. 2012;2(2):65-74.

13. Ferguson MA, Alderson NL, Trost SG, Essig DA, Burke JR, Durstine JL. Effects of four different single exercise sessions on lipids, lipoproteins, and lipoprotein lipase. J Appl Physiol (1985). 1998;85(3):1169-74.

14. Kantor MA, Cullinane EM, Sady SP, Herbert PN, Thompson PD. Exercise acutely increases high density lipoprotein-cholestero and lipoprotein lipase activity in trained and untrained men. Metabolism. 1987;36(2):188-92.

15. Behaeein B, Yadolazadeh A, Same H, Etemadi S, Sadegi H, Salehiyan $\mathrm{MH}$. Relation of anthropometric characteristics in women with diffrent types of MS and Comparison with Health ones. Annals Biol Res. 2011;2(5):14-21.

16. Dionyssiotis Y. Body composition in multiple sclerosis. Hippokratia. 2013;17(1):7-11.

17. Lambert CP, Lee Archer R, Evans WJ. Body composition in ambulatory women with multiple sclerosis. Arch Phys Med Rehabil. 2002;83(11):1559-61.

18. Poulain M, Doucet M, Major GC, Drapeau V, Series F, Boulet LP, et al. The effect of obesity on chronic respiratory diseases: pathophysiology and therapeutic strategies. CMAJ. 2006;174(9):1293-

19. Dalgas U, Stenager E. Exercise and disease progression in multiple sclerosis: can exercise slow down the progression of multiple sclerosis? Ther Adv Neurol Disord. 2012;5(2):81-95.

20. Romberg A, Virtanen A, Ruutiainen J, Aunola S, Karppi SL, Vaara $\mathrm{M}$, et al. Effects of a 6-month exercise program on patients with multiple sclerosis: a randomized study. Neurology. 2004;63(11):2034-8.

21. fayazi B, Parnow A, Ahsan B, Yousefvand Z. The effect of aerobic training on functional capacity and percent body fat in women with moderate multiple sclerosis. Arak Univ of Med Sci J. 2013;16(1):87-97.

22. Kin Isler A, Kosar SN, Korkusuz F. Effects of step aerobics and aerobic dancing on serum lipids and lipoproteins. J Sports Med Phys Fitness. 2001;41(3):380-5.

23. Jaywant PJ. Effect of Aerobic Dance on the Body Fat Distribution and Cardiovascular Endurance in Middle Aged Women.J Exercise Sci Physiother. 2013;9(1):6-10.

24. Kostic R, Duraskovic R, Miletic D, Mikalacki M. Changes In The Cardiovascular Fitness And Body Composition Of Women Under The Influence Of The Aerobic Dance. Phys Educ Sport. 2006;4(1):59-71

25. Ebrahimi Atri A, Sokhangoi M, Sarvari F. The comparison of resistance and endurance exercises on fatigue severity and balance in women with multiple sclerosis. J Sport Med. 2013;5(1):89102

26. Friedewald WT, Levy RI, Fredrickson DS. Estimation of the concentration of low-density lipoprotein cholesterol in plasma without use of the preparative ultracentrifuge. Clin Chem. 1972;18(6):499-502.

27. Hoffman J. Norms for fitness, performance, and health. 1st ed: Human Kinetics; 2006.

28. Wilmore JH, Costill DL. Physiology of sport and exercise.: Human
Kinetics Publishers; 1994.

29. Roubenoff R, Rall LC. Humoral mediation of changing body composition during aging and chronic inflammation. Nutr Rev. 1993;51(1):1-11.

30. El Maghraoui A, Borderie D, Cherruau B, Edouard R, Dougados M, Roux C. Osteoporosis, body composition, and bone turnover in ankylosing spondylitis. J Rheumatol.1999;26(10):2205-9.

31. Giubilei F, Antonini G, Di Legge S, Sormani MP, Pantano P, Antonini R, et al. Blood cholesterol and MRI activity in first clinical episode suggestive of multiple sclerosis. Acta Neurol Scand. 2002;106(2):109-12.

32. Petajan JH, Gappmaier E, White AT, Spencer MK, Mino L, Hicks RW. Impact of aerobic training on fitness and quality of life in multiple sclerosis. Ann Neurol. 1996;39(4):432-41.

33. Schapiro RT, Petajan JH, Kosich D, Molk B, Feeney J. Role of Cardiovascular Fitness in Multiple Sclerosis: A Pilot Study. Neurorehabil Neural Repair. 1988;2(2):43-9.

34. Plotnikoff RC, Courneya KS, Trinh L, Karunamuni N, Sigal RJ. Aerobic physical activity and resistance training: an application of the theory of planned behavior among adults with type 2 diabetes in a random, national sample of Canadians. Int $J \mathrm{Be}-$ hav Nutr Phys Act. 2008;5:61.

35. Van Gaal L, Rillaerts E, Creten W, De Leeuw I. Relationship of body fat distribution pattern to atherogenic risk factors in NIDDM. Preliminary results. Diabetes Care. 1988;11(2):103-6.

36. Despres JP, Moorjani S, Tremblay A, Poehlman ET, Lupien PJ Nadeau A, et al. Heredity and changes in plasma lipids and lipoproteins after short-term exercise training in men. Arteriosclerosis. 1988;8(4):402-9.

37. Marsh H, Alexander J, Costello E. Short-term exercise program effect on physical work capacity. Arch Phys Med Rehabil. 1986;67:644.

38. Gutin B, Barbeau P, Owens S, Lemmon CR, Bauman M, Allison I, et al. Effects of exercise intensity on cardiovascular fitness, tota body composition, and visceral adiposity of obese adolescents. Am J Clin Nutr. 2002;75(5):818-26.

39. Ronti T, Lupattelli G, Mannarino E. The endocrine function of adipose tissue: an update. Clin Endocrinol (Oxf). 2006;64(4):35565.

40. Franklin SS. Arterial stiffness and hypertension: a two-way street? Hypertension. 2005;45(3):349-51.

41. Krupa D. Aerobic Exercise Reduces Risk Of Coronary Artery Disease In People With Mild/Moderate MS. APS Press Room. 2007;202:249-4174.

42. Lokey EA, Tran ZV. Effects of exercise training on serum lipid and lipoprotein concentrations in women: a meta-analysis. In J Sports Med.1989;10(6):424-9.

43. Cuff DJ, Meneilly GS, Martin A, Ignaszewski A, Tildesley HD, Frohlich JJ. Effective exercise modality to reduce insulin resistance in women with type 2 diabetes. Diabetes Care 2003;26(11):2977-82

44. Kraus WE, Houmard JA, Duscha BD, Knetzger KJ, Wharton MB, McCartney JS, et al. Effects of the amount and intensity of exercise on plasma lipoproteins. N Engl J Med. 2002;347(19):1483-92.

45. Bahrami A, Saremi A. Effect of caloric restriction with or without aerobic training on body composition, blood lipid profile, insulin resistance, and inflammatory marker in middle-age obese/overweight men. Arak Med Univ J. 2011;14(3):11-9.

46. Formica CA, Cosman F, Nieves J, Herbert J, Lindsay R. Reduced bone mass and fat-free mass in women with multiple sclerosis: effects of ambulatory status and glucocorticoid Use. Calcif Tissue Int. 1997;61(2):129-33. 